\title{
A Simultaneous-Imaging Machine-Vision Approach for the Precision Alignment of Two mm-Wave Antennas
}

\author{
Joshua A. Gordon, David R. Novotny \\ National Institute of Standards and Technology, Electromagnetics Division, Boulder, CO 80305 USA
}

\begin{abstract}
We present an optical imaging tool, the Overlay Imaging Aligner (OIA), developed to aid in the mechanical alignment of antenna components in the mm-wave and low- $\mathrm{THz}$ frequency regimes $(50-500 \mathrm{GHz})$ where wavelength size poses significant challenges for alignment. A polarization-selective machine-vision approach used to generate two simultaneous overlaid images along a common axis allows for aligning two antenna components to within fractions of a wavelength. The concept and application examples are presented along with a quantitative assessment of the alignment accuracy in the WR-2.2 band where the OIA alignment is compared to an electrical alignment.
\end{abstract}

Index Terms - alignment, antenna, machine vision, mm-wave, terahertz, waveguide

\section{INTRODUCTION}

$\mathrm{I}^{\mathrm{N}}$ $\mathrm{N}$ recent years more and more systems e.g. [1-4] are being developed in the mm-wave and terahertz frequency ranges. At these high frequencies $(50-500 \mathrm{GHz})$, wavelengths can approach sub-millimeter dimensions. With such wavelength scales, the mechanical alignment of waveguide and antenna components becomes increasingly difficult. Antenna characterization techniques such as those used in extrapolation measurements [3,5], near field measurements [6-9] and general spatial antenna characterization require the precise positioning of antenna components. At these frequencies, new techniques must be developed for achieving the same alignment tolerances that has traditionally been straightforward to achieve in the $\mathrm{MHz}$ and lower $\mathrm{GHz}$ regimes. Although other alignment approaches using laser tracking systems have been previously proposed [10], in this letter we present a compact optical alignment tool that utilizes polarization-selective imaging to simultaneously capture and align, in real-time, two digital images of antenna components along a common axis, to within sub-wavelength precision and accuracy.

\section{MOTIVATION AND CONCEPT}

At the mm-wave and $\mathrm{THz}$ frequencies, the wavelength approaches $\mathrm{mm}$ to sub-mm dimensions. The resulting mechanical tolerances and position accuracy of mounts and alignment stages must be significantly better than half a wavelength, depending on accuracy requirements; i.e. tens to hundreds of microns.

An optical imaging tool using a polarization-selective machine-vision approach was developed at NIST-Boulder, CO

U.S. Government work not protected by U.S. copyright. to address these alignment issues. This tool, the Overlay Imaging Aligner (OIA) provides real-time alignment of two waveguide or antenna components as well as mm-wave focusing and beam- shaping optics along a common axis, by overlaying simultaneous digital images of both components on a common imaging detector array.

The real-time simultaneous imaging of two antenna components is accomplished by generating two polarized images from the (assumed) unpolarized scene of the ambient or actively illuminated antennas. The OIA prototype system was designed to accommodate a field-of-view (FOV) suitable for aligning mm-wave antenna components. The optical components were chosen to obtain a transverse alignment precision of, $\Delta x=52 \mu \mathrm{m}$, which, at an operating frequency of $300 \mathrm{GHz}$, provides a precision of $\approx \lambda / 20$.

\section{ANTENNA COMPONENT ALIGNMENT}

\section{A. Horn Antenna Alignment}

The alignment images of two WR-03 pyramidal horns obtained with the OIA are shown in Fig. 1a, 1b. The alignment error is clearly visible in Fig. 1a, where the aperture of the horns, flanges, and precision alignment pins do not overlap. Figure $1 \mathrm{~b}$ shows the antennas after adjusting the antanna mounts, where the alignment of the two antennas is clearly visible. The azimuth and elevation alignment of these horns was achieved by aligning multiple planes of the horns to the same axis.
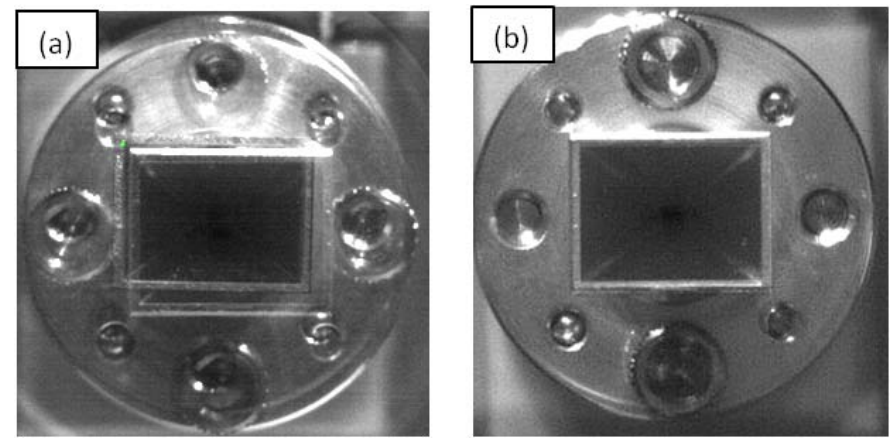

Fig. 1. OIA image of two WR-03 pyramidal antennas showing, (a) overlapped misaligned horn images, and (b) horns after alignment where the image now appear as one single horn.

\section{B. Lens Alignment to Horns}

Precision lens alignment is achieved by imaging the horns through the optically clear TPX lenses with the OIA. The TPX lenses will act as magnifying glasses and produce magnified virtual images of the horn apertures. These virtual horn images act as virtual objects for the OIA optics, and can be re- 
imaged by the OIA optics. Any misalignment of the lenses axes relative to the horn axes will appear as these virtual horns being misaligned.
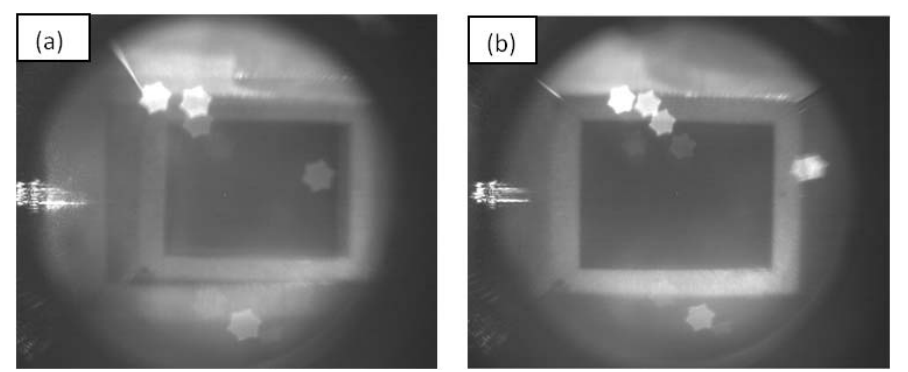

Fig. 2. (a) Misalignment of "virtual horns" seen through TPX lenses with the OIA. (b) Both "virtual horns" are aligned and appear as overlapped and indistinguishable in the OIA image after lens alignment.

It is then a matter of aligning these virtual horns by adjusting the TPX lenses and not the actual horns. Figures $2 \mathrm{a}, 2 \mathrm{~b}$ show the positions of the virtual horns before and after lens alignment.

A comparison of the OIA alignment to an electrical alignment was also made. The transmit horn position $\mathrm{T}_{\mathrm{x}}$ was moved in $10 \mu \mathrm{m}$ steps over a range of $+/-200 \mu \mathrm{m}$, with $0 \mu \mathrm{m}$ referencing the position that had been determined by alignment using only the OIA. The value of $\mathrm{T}_{\mathrm{x}}$ that was needed to peak the power out of the receiver gives a measure of how far off from peaked electrical alignment the OIA achieves. Data for frequencies, $380 \mathrm{GHz}, 400 \mathrm{GHz}$, and $450 \mathrm{GHz}$ are shown in Fig. 3 where $|\mathrm{S} 12|$ has been normalized by $|\mathrm{S} 12|$ at $T_{x}=0 \mu \mathrm{m}$. These data show extremely good alignment accuracy obtained with the OIA, with errors in the relative peak electrical alignment transmitted power $\ll 0.1 d B$ and discernable antenna position errors of $\Delta x \approx 50 \mu \mathrm{m}$, as shown by the vertical dotted line in Fig 3 .

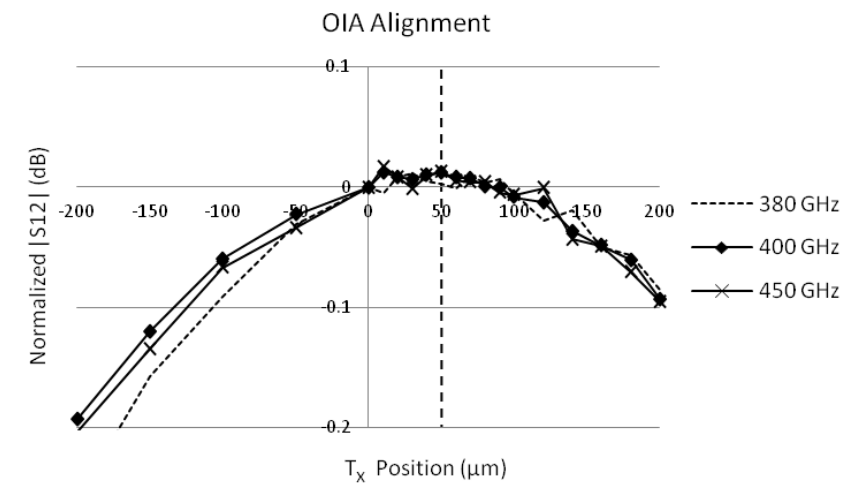

Fig. 3. Receive power normalized to the OIA alignment position at $0 \mu \mathrm{m}$ as a function of transmit horn position $\mathrm{T}_{\mathrm{x}}$ for frequencies, $380 \mathrm{GHz}, 400 \mathrm{GHz}$, and $450 \mathrm{GHz}$. Peak electrical alignment position is shown by the vertical dotted line.

\section{CONCLUSION}

In this paper, we presented an optical imaging tool, the Overlay Imaging Aligner (OIA), developed at NIST-Boulder, $\mathrm{CO}$, to aid in the alignment of antenna components in the $\mathrm{mm}$ - wave and low-THz frequency regimes. The OIA uses a polarization-selective machine vision approach to overlay two simultaneous real-time images along a common axis, for aligning two antenna components to within fractions of a wavelength. The concept and application examples of the alignment of two WR-03 pyramidal horn antennas, as well as the alignment of a two WR-2.2 horn and TPX lens system are presented. Data over the $380-450 \mathrm{GHz}$ range comparing the OIA alignment to an electrical alignment is presented demonstrating nominally obtainable transverse alignment accuracies of $\Delta x \approx 50 \mu \mathrm{m}$ (i.e., $\lambda / 16$ to $\lambda / 13$ ).

\section{ACKNOWLEDGEMENTS}

The authors thank The Department of Homeland Security Science and Technology Directorate for sponsoring this work under IAA \# HSHQDC-10-X-00452 with the National Institute of Standards and Technology.

\section{REFERENCES}

[1] Charles R. Dietlein, Jon E. Bjarnason, Erich N. Grossman and Zoya Popović, "Absorption, transmission, and scattering of expanded polystyrene at millimeter-wave and terahertz frequencies", Proc. SPIE 6948, 69480E (2008)

[2] R. H. Direen, D. Novotny, K. MacReynolds, "Active Millimeter-Wave Imaging: Detection of Concealed Weapons", AMTA Proceedings, pp. 287-289, October 2010, Atlanta Georgia

[3] M. Ameya, M. Hirose, S. Kurokawa, "W-band Antenna Gain Calibration Extrapolation Using Time-Domain Gating", AMTA Proceedings, pp. 248-253, November 2009, Salt Lake City Utah.

[4] D. Cimini, E. R. Westwater, A. J. Gasiewski, M. Klein, V. Y. Leuski, S. G. Dowlatshahi,"The Ground Based Scanning Radiometer: A Powerful Toll for Study of the Arctic Atmosphere", IEEE Trans. Geosci. Remote. Sens., vol. 45, no. 9, pp. 2759-2777, 2007

[5] A. G. Repjar, A. C. Newell, D. T. Tamura, "Extrapolation Range Measurements for Determining Antenna Gain and Polarization", NBS Tech Note 1311, August 1987

[6] A. D. Yaghjian, "Near Field Antenna Measurments On A Cylindrical Surface: A Source Scattering-Matrix Formulation", NBS Tech Note 696, September 1977.

[7] R. C. Wittmann, C. F. Stubenrauch, "Spherical Near-Field Scanning: Experimental and Theoretical Studies", NISTIR 3955, July 1990.

[8] R. C. Wittman, B. K. Alpert, M. H. Francis, "Near-Field Antenna Measurments Using Nonideal Measurement Locations", IEEE Trans. Antenn. Prop. Vol. 46, no. 5, 1998.

[9] A. D. Yaghjian, "An Overvie of Near-Field Antenna Measurement", IEEE Trans. Antenna. Prop. vol. AP-34, no. 1.1986.

[10]T. Proctor, "An Approach to Antenna Range Alignment Utilizing a Laser Tracking System", AMTA Proceedings, pp. 324-329, October 2010, Atlanta Georgia 